\title{
MITTEILUNGEN
}

\section{„Der Bundestag verhandelt öffentlich“ (Art. 42 GG) - Zur Notwendig- keit eines Parlamentskanals. Eine Veranstaltung der Deutschen Vereini- gung für Parlamentsfragen in Berlin am 29. November 2007}

Über die Notwendigkeit eines Parlamentskanals wurde zuletzt vor 30 Jahren gestritten und seither mitunter diskutiert. Norbert Lammert, schon damals Mitglied des Bundestages und heute dessen Präsident, belebte die Diskussion im vergangenen Jahr wieder ${ }^{1}$, und traf dabei sowohl auf Wohlwollen als auch auf Kritik. Der kontroversen Thematik widmete sich die Deutsche Vereinigung für Parlamentsfragen in einer Veranstaltung am 29. November 2007; am selben Tag hatte Lammert seine Gedanken zu einem möglichen Parlamentsfernsehen auch im Ältestenrat des Bundestages vorgetragen und war nach eigener Aussage auf "große Sympathie" gestoßen.

Der Bundestagspräsident sah in seinem Einführungsreferat eine zeitliche Parallele zwischen den Anfängen des Fernsehens und des Parlamentarismus in der Bundesrepublik. Schon die konstituierende Sitzung des ersten Bundestages am 7. September 1949 wurde direkt vom Rundfunk übertragen, die erste Fernsehaufnahme einer Bundestagsdebatte folgte am 19. März 1953. Die Einführung des Privatfernsehens brachte eine grundlegende Veränderung mit sich, die sich unter anderem auf die Übertragung der Debatten auswirkte und langfristig auch in einen eigenen Spartenkanal mit dem Namen Phoenix mündete. Die Entscheidung für diesen Kanal wurde, so Lammerts These, allerdings mit einem hohen Preis bezahlt, nämlich mit der „Auswanderung der Fernsehberichterstattung (über das Parlament) aus dem öffentlich-rechtlichen Rundfunk“. Dies sei „die reine Wahrheit“. Zwar sei die Berichterstattung von Phoenix ,außerordentlich gut gelungen“, aber nicht vollständig: Circa 50 Prozent der Aktivitäten des Bundestages blieben unübertragen, Ausschusssitzungen würden überhaupt nicht gesendet.

Lammert sah vor diesem Hintergrund eine prinzipielle und eine praktische Problematik: Für erstere gäbe es eine unmissverständliche Verfassungslage, nach der die Verhandlungen des Bundestages öffentlich zu sein haben. Heutzutage seien keine technischen Hindernisse und Restriktionen mehr vorhanden. Zudem werde ohnehin alles im Bundestag aufgezeichnet. Das Angebot sei also vorhanden, es werde nur nicht zur Übertragung genutzt. Praktisch ergäbe sich das Problem, dass ein Parlamentsfernsehen nicht so arbeiten könne wie Phoenix. Letztlich besteht deshalb ein Dilemma: Einerseits hält Lammert den Einsatz des öffentlich-rechtlichen Fernsehens in der Parlamentsberichterstattung für nicht überzeugend und nicht akzeptabel, andererseits überzeugt ihn das Angebot, das der Bundestag selbst machen könnte, um dieses Manko zu kompensieren, ebenfalls nicht.

1 Auch eine Studie wurde im Auftrag des Bundestages erstellt: Hubertus Gersdorf, Parlamentsfernsehen des Deutschen Bundestages, Berlin 2007. 
Auf dem Podium setzten sich zwei Vertreter der Medien und zwei Bundestagsabgeordnete mit der Thematik und den Ausführungen des Bundestagspräsidenten auseinander. Christoph Minhoff, Programm-Geschäftsführer von Phoenix, verteidigte seinen Sender. Erstens habe nicht dieser seinen Programmauftrag verändert, sondern die Politik, als aus dem Projekt eines europäischen Ereignis- und Parlamentskanals der Ereignis- und Dokumentationskanal Phoenix wurde. Zweitens berichte sein Sender mehr aus dem Bundestag, als Lammert berücksichtigt habe; auch Ausschusssitzungen würden sehr wohl übertragen. Drittens arbeite Phoenix ohne Quotenziel. Häufig wird nur ein Marktanteil von 0,1 Prozent, was circa 10.000 Zuschauern entspricht, erreicht; Highlights bilden zum Beispiel Haushaltsdebatten, die auf 1,4 bis 1,8 Prozent Marktanteil und damit auf eine Reichweite von circa 80.000 Zuschauer kommen. Außerdem könne Phoenix keine Öffentlichkeitsarbeit des Bundestages leisten, seine Aufgabe bestehe in der kritischen journalistischen Begleitung des Parlaments. Ein eigener Parlamentskanal würde hingegen das Problem eines Doppelangebots mit Phoenix und die berechtigte Frage nach dem Einsatz von Gebührengeldern aufwerfen. Zudem würde solch ein Kanal, wenn er - wie Pläne erkennen ließen - über digitale Satelliten verbreitet werden soll, nur 25 Prozent der Bevölkerung erreichen. Warum, so endete Minhoff, sollte man sich stattdessen nicht stärker auf das Internet, in dem das Parlament rund um die Uhr gezeigt werden kann, konzentrieren?

Für Tissy Bruns vom Berliner Tagesspiegel würde die Errichtung eines Parlamentskanals unweigerlich zur „Aussonderung der Parlamentsberichterstattung“ aus Phoenix führen. Sie sah eine dramatische Entwicklung, die die Berichterstattung über Parlamente erheblich gewandelt habe. Zwei Hauptveränderungen haben ihr zufolge stattgefunden: die früher strukturierte Öffentlichkeit, auch in Form einer Fernsehnation, sei einer zersplitterten und zerstreuten Öffentlichkeit gewichen, und die zuvor fast eheliche Beziehung zwischen Politik und Medien in die Brüche gegangen. Die Frage eines Parlamentskanals sollte daher auch in einen größeren Rahmen gesetzt und mit der Frage verknüpft werden, wo Abgeordnete, Politiker und politische Journalisten überhaupt noch Möglichkeiten haben, „ihre Stimme im öffentlichen Raum wieder deutlicher zu machen“. Die Frage, die alle Anwesenden der Veranstaltung eine, sei ohnehin, wie die Bevölkerung für das Politische zu interessieren ist.

Die Frage der Transparenz stellte der SPD-Bundestagsabgeordnete Ulrich Kelber in den Vordergrund, zu der für ihn auch die Einordnung und journalistische Einbettung des Berichteten gehört. Eine reine Übertragung könne nicht genügen. Mit Blick auf die weitere Verbreitung von Breitbandanschlüssen warb er für die Möglichkeiten des Internets, in dem Ausschusssitzungen auch parallel und als „video on demand“ angeboten werden können. Bessere Antworten des Bundestages auf die gegenwärtige Situation als ein eigener Parlamentskanal wären ihm zufolge, erstens das Internetangebot weiter auszubauen, zweitens das Parlament selbst zu verbessern, so dass das Angebot an die Öffentlich-Rechtlichen attraktiver werde, und drittens den Druck auf die öffentlich-rechtlichen Anstalten für mehr Berichterstattung zu erhöhen. Kelber trat für eine „ungefilterte“ direkte Berichterstattung via Internet und für eine eingebettete wie kommentierte im herkömmlichen Medium des Fernsehens ein.

Der FDP-Bundestagsabgeordnete Hans-Joachim Otto verneinte die Frage, ob die Einrichtung eines Parlamentskanals verfassungsrechtlich geboten sei, denn „Öffentlichkeit heißt (...) nicht, dass es jedem sozusagen vor die Tür getragen wird“. Die Tendenz zur Verspartung monierte er ebenso wie Lammert und Bruns und plädierte dafür, die Anstrengungen darauf zu konzentrieren, das Parlament wieder verstärkt in die Hauptprogramme zu 
bekommen. Dort gäbe es heute eine totale Fixierung auf Minister. So beklagte er sich auch weniger über die fehlenden 50 Prozent bei Phoenix als über die fehlenden 95 Prozent bei ARD und ZDF. Da Phoenix ein gutes Programm mache, mindere dies die Notwendigkeit eines Parlamentskanals via Kabel oder Satellit. Wie Kelber hielt auch Otto eine Kommentierung und Einordnung des Berichteten für notwendig, wodurch gleichzeitig das verfassungsrechtliche Problem des Staatsfernsehens berührt werde. So trat auch er für die Internetangebote des Bundestages und von Phoenix ein, blieb aber skeptisch gegenüber Ausdehnungen in Form eines eigenen Kanals.

Lammert berief sich in seiner Erwiderung darauf, kein Lösungskonzept vorgetragen, sondern Defizite beschrieben zu haben. Der derzeitige Zustand der Parlamentsberichterstattung sei eben nicht akzeptabel, dies sollte vor allem verändert und verbessert werden.

In der allgemeinen Diskussion meldeten sich ebenfalls Journalisten wie Abgeordnete zu Wort. Auch hier überwog wie auf dem Podium die Ablehnung eines Parlamentskanals. Reinhard Grindel, MdB aus der CDU/CSU-Fraktion, schlug stattdessen vor, im Funktionsauftrag der öffentlich-rechtlichen Sender mehr Parlamentsberichterstattung zu verankern. Peter Frey, Leiter der Parlamentsredaktion des ZDF, trat den Vorwürfen gegen die öffentlich-rechtlichen Sender entgegen und verwies auf ihre großen Parlamentsredaktionen in Berlin mit zahlreichen Korrespondenten und deren kontinuierliche Arbeit für die Nachrichtensendungen. Zudem gäbe es heute einen Markt, den die Politik durch die Einführung des Privatfernsehens selbst geschaffen habe. Auch müsste der Bundestag, wie Kelber aus Abgeordnetensicht kritisch gefordert hatte, selbst stärker darüber nachdenken, sich interessanter zu machen - auch in Form von kontroversen Debatten in hoher sprachlicher Qualität. In dieser Hinsicht übten auch andere Abgeordnete Selbstkritik: Otto, Lammert und Josef Winkler (Fraktion Bündnis 90/Die Grünen) sahen hier Raum für Verbesserungen. Auf journalistischer Seite wurde hingegen geradezu der Schulterschluss mit der Politik gesucht, denn letztlich eine, so beispielsweise Frey, beide das gemeinsame Anliegen, die Bürger zu erreichen und zu interessieren. Zwar haben beide Seiten nicht die gleiche Aufgabe, so Minhoff, aber das gleiche Interesse, den Parlamentarismus in Deutschland zu stärken.

Verwundert zeigte sich Tino Kunert, Mitglied des RBB-Rundfunkrates, über den Befund, die Parlamentsberichterstattung in den öffentlich-rechtlichen Sendern sei nicht akzeptabel. In keinem der Landesrundfunkräte, die ja die Aufgabenerfüllung der Sender zu beurteilen und zu verantworten haben, wurde eine solche Debatte bisher angestoßen, auch nicht von Vertretern der CDU. Georg-Paul Hefty entwarf als Zukunftsszenario, dass mit der Einführung eines Parlamentskanals für den Bundestag auch die Landesparlamente und das Europäische Parlament zu dieser Möglichkeit greifen würden. Welcher Gewinn ließe sich aber aus der Präsenz von 17 oder 18 Parlamentskanälen ziehen? Einen höheren Erkenntnisgewinn für die Bürger bezweifelte er. Nils Diederich warnte vor einem Parlamentskanal als eigene ,journalistische Agentur“, in der Politik und Journalismus vermischt würden. Das Problem sei vielleicht nicht verfassungsrechtlich relevant, verfassungspolitisch jedoch auf jeden Fall.

Unterstützung erhielt Lammert von Josef Winkler und Wolfgang Zeh, Direktor beim Bundestag a.D. Winkler begrüßte Lammerts Anliegen als richtig und trat für seine Umsetzung ein. Eine Verbreitung nur via Internet kritisierte er, da auch hier aufgrund von DSL-freien Gegenden keine hundertprozentige Abdeckung zu erreichen ist. Zudem seien 5.000 oder 10.000 Zuschauer immer noch mehr als diejenigen, die zufällig eine halbe Stunde auf der Besuchertribüne des Bundestages sitzen. Zeh sah in Art. 5 GG kein Problem, da das Parla- 
ment nicht gemeint sein könne, wenn von Staatsfernsehen die Rede ist. Das Parlament bilde die Gesellschaft ab und sei nicht als Staat im Sinne von Art. 5 GG zu verstehen. Daher könne dieser Grundgesetzartikel auch eher als Verpflichtung für das Parlament auf ein Fernsehangebot interpretiert werden.

Die Veranstaltung entwickelte sich zu einer generellen Debatte über das Verhältnis von Politik und Medien, wobei beide Seiten durchaus unterschiedliche Schwerpunkte setzten: Auf der Seite der Abgeordneten wurde die Unzufriedenheit Lammerts mit der derzeitigen Berichterstattung über den Bundestag im Großen und Ganzen geteilt. Als Alternativen zu einem Parlamentskanal wurden eine verstärkte Konzentration auf das Internet und eine verbesserte Berichterstattung im öffentlich-rechtlichen Fernsehen befürwortet, wobei für letztere Möglichkeit auch die Verbesserung des eigenen parlamentarischen Angebots im Sinne von gesteigerter Attraktivität angemahnt wurde. Die journalistischen Vertreter, insbesondere der elektronischen Medien, waren hingegen nicht prinzipiell unzufrieden mit der Berichterstattung, stattdessen verwiesen sie auf das generelle Problem, die Bürger für Politik zu interessieren, und suchten in diesem Sinne eine gemeinsame Ebene mit den Abgeordneten für Bemühungen um das Politische, das politische Interesse und den Parlamentarismus; darin sahen sie sich „in einem Boot“ mit den Parlamentariern. Auf beiden Seiten galten für die Installierung eines eigenen Kanals das Doppelangebot, insbesondere mit Phoenix, und der Bedarf an journalistischer Kommentierung und Einordnung, die nicht ohne Aufwand zu leisten wären, als problematisch.

In der Sache zeigte sich Lammert am Ende der Diskussion „sehr ermutigt“. So bemühte er sich auch in den folgenden Monaten: Zuletzt stellte er sein Anliegen am 5. März 2008 im Bundestagsausschuss für Kultur und Medien vor. Dieser unterstützte den Bundestagspräsidenten in seiner Forderung nach mehr Parlamentsberichterstattung im öffentlichrechtlichen Fernsehen; die Idee eines eigenen Parlamentskanals wurde aber auch dort kontrovers aufgenommen. ${ }^{2}$ Inzwischen haben ARD und ZDF Versäumnisse eingestanden und mehr Berichterstattung, zumindest von Sonderveranstaltungen, in Aussicht gestellt.

Franziska Höpcke

2 Vgl. Sandra Ketterer, Pro und contra Parlamentsfernsehen, in: Das Parlament vom 10. März 2008. 